OPEN ACCESS

Edited by:

Ying $X u$,

The State University of New York at

Buffalo, USA

Reviewed by: Ramesh Kandimalla, Emory University, USA Neha Sehgal,

Wisconsin Institute for Discovery,

USA

${ }^{*}$ Correspondence:

Dinggang Shen

dinggang_shen@med.unc.edu;

Shifu Xiao

xiaoshifu@msn.com

tThese authors have contributed equally to this work.

Received: 21 February 2016 Accepted: 29 April 2016

Published: 18 May 2016

Citation:

Wang T, Shi F, Jin Y, Jiang W, Shen D and Xiao $S$ (2016) Abnormal

Changes of Brain Cortical Anatomy and the Association with Plasma MicroRNA107 Level in Amnestic Mild

Cognitive Impairment.

Front. Aging Neurosci. 8:112. doi: 10.3389/fnagi.2016.00112

\section{Abnormal Changes of Brain Cortical Anatomy and the Association with Plasma MicroRNA107 Level in Amnestic Mild Cognitive Impairment}

\author{
Tao Wang ${ }^{1,2,3 \dagger}$, Feng Shi ${ }^{3 \dagger}$, Yan $\mathrm{Jin}^{3}$, Weixiong Jiang ${ }^{3}$, Dinggang Shen ${ }^{3,4 *}$ \\ and Shifu Xiao ${ }^{1,2 *}$ \\ ${ }^{1}$ Department of Geriatric Psychiatry, Shanghai Mental Health Center, Shanghai Jiao Tong University School of Medicine, \\ Shanghai, China, ${ }^{2}$ Alzheimer's Disease and Related Disorders Center, Shanghai Jiao Tong University, Shanghai, China, \\ ${ }^{3}$ IDEA Lab, Department of Radiology and BRIC, University of North Carolina at Chapel Hill, Chapel Hill, NC, USA, \\ ${ }^{4}$ Department of Brain and Cognitive Engineering, Korea University, Seoul, South Korea
}

MicroRNA107 (Mir107) has been thought to relate to the brain structure phenotype of Alzheimer's disease. In this study, we evaluated the cortical anatomy in amnestic mild cognitive impairment (aMCl) and the relation between cortical anatomy and plasma levels of Mir107 and beta-site amyloid precursor protein (APP) cleaving enzyme 1 (BACE1). Twenty aMCl $(20 \mathrm{aMCl})$ and 24 cognitively normal control (NC) subjects were recruited, and T1-weighted MR images were acquired. Cortical anatomical measurements, including cortical thickness (CT), surface area (SA), and local gyrification index (LGI), were assessed. Quantitative RT-PCR was used to examine plasma expression of Mir107, BACE1 mRNA. Thinner cortex was found in aMCl in areas associated with episodic memory and language, but with thicker cortex in other areas. SA decreased in aMCl in the areas associated with working memory and emotion. LGI showed a significant reduction in $\mathrm{aMCl}$ in the areas involved in language function. Changes in Mir107 and BACE1 messenger RNA plasma expression were correlated with changes in CT and SA. We found alterations in key left brain regions associated with memory, language, and emotion in aMCl that were significantly correlated with plasma expression of Mir107 and BACE1 mRNA. This combination study of brain anatomical alterations and gene information may shed lights on our understanding of the pathology of AD.

Clinical Trial Registration: http://www.ClinicalTrials.gov, identifier NCT01819545.

\footnotetext{
Keywords: Alzheimer's disease, amnestic mild cognitive impairment, genetics, biological markers, surface-based morphometry
}

\section{INTRODUCTION}

Alzheimer's disease $(\mathrm{AD})$ is characterized by a progressive decline in cognition and daily function. It is the most common form of dementia worldwide. Pathologically, AD is defined by the intracellular accumulation of aggregated and hyperphosphorylated tau protein, the extracellular deposition of amyloid $\beta$ ( $\mathrm{A} \beta$ ) peptides, and the accumulation of neurofibrillary tangles and amyloid plaques throughout the cortex (De Strooper, 2010; Kandimalla et al., 2013a,b). Amnestic mild cognitive impairment (aMCI) is thought to be a predementia phase of AD (Albert et al., 2011), and is characterized by the same etiology to a lesser degree (Petersen et al., 2006). 
A widely accepted hypothesis links the major pathology of $\mathrm{AD}$ to the generation and subsequent accumulation of $A \beta$ through sequential cleavage of amyloid precursor protein (APP) by betasite APP cleaving enzyme $1(\mathrm{BACE} 1)^{1}$ and $\gamma$-secretase (Dislich and Lichtenthaler, 2012). Regulation of expression of the proteins involved in this process plays an important role in $\mathrm{AD}$ (Bettens et al., 2009).

BACE1 expression is regulated by BACE1 messenger RNA (BACE1 mRNA), while BACE1 mRNA is regulated by microRNA (Mir/miRNA). Accumulating evidence suggests that alterations in Mirs contribute to AD (Sathya et al., 2012). Studies have found decreases in Mirs in postmortem brain of $\mathrm{AD}$ patients, as compared to normal controls (NCs). More interestingly, Mir expressions are altered not only in the brains of $\mathrm{AD}$ patients, but also in their cerebral spinal fluid (CSF) and blood plasma (Hébert et al., 2010). Among these, MicroRNA107 (Mir107) ${ }^{2}$ targets genes directly related to $\mathrm{AD}$, including BACE1. In microarray studies, Mir107 levels were substantially reduced in the temporal cortex of $\mathrm{AD}$ patients (Wang et al., 2011).

The accumulation of neurofibrillary tangles and amyloid plaques in the cortex is associated with gray matter (GM) atrophy and volume decline. Abnormal brain anatomy has been identified as an important feature of the pathophysiological process of $\mathrm{AD}$, and can be visualized using different modalities of MRI for GM (Meda et al., 2013) and white matter (Li et al., 2013; Jin et al., 2015; Wang et al., 2016), respectively. Those features can be accurately used to differentiate $\mathrm{AD}$ patients from the normally aging population (Zhan et al., 2014, 2015). Morphological parameters have been widely used to detect brain abnormalities in aMCI. Previous voxel-based morphometry (VBM) studies reported that aMCI subjects showed GM atrophy in entorhinal cortex, posterior cingulate cortex, and medial prefrontal cortex (Apostolova et al., 2007). Although VBM is valuable in measuring morphological changes in $\mathrm{AD}$ patients, it does not capture cortical sulcal and gyral patterns, or their changes due to the disease (Davatzikos, 2004).

Surface-based morphometry (SBM) is one approach that captures subtle cortical surface changes, and can examine cortical thickness (CT) and surface area (SA) of GM separately. Studies have reported that aMCI patients showed overall cortical thinning and sulcal widening, compared to NCs (Davatzikos, 2004). SBM can also assess the degree of cortical folding using local gyrification index (LGI; Im et al., 2008; Li et al., 2014a). These measurements provide unique and complementary information that reflects distinct cortical properties ( $\mathrm{Li}$ et al., 2014b; Libero et al., 2014). Investigation of these surface-based measures can provide information beyond those volumetric abnormalities previously uncovered in AD. By measuring CT, SA, and LGI, we may uncover the underlying changes in cortical architecture of AD (Libero et al., 2014).

A definite diagnosis of $\mathrm{AD}$ is determined by postmortem examination. Currently, amyloid PET examination (Fleisher et al., 2012) can be utilized for early diagnosis, but, because PET

\footnotetext{
${ }^{1}$ http://www.informatics.jax.org/marker/MGI:1346542

${ }^{2}$ http://www.informatics.jax.org/marker/MGI:3619063
}

examination is expensive, a routine diagnosis of aMCI and AD still depends on combination of clinical and neuropsychological tests. Therefore, there is great interest in identifying $\mathrm{AD}$ genotype and phenotype associated biomarkers in brain anatomy and plasma.

Our goal in this study is to investigate alterations in cortical anatomy in aMCI, as well as to detect any relationship between structural changes and neuropsychological scores, levels of Mir107, and BACE1 mRNA in plasma. The findings of this study will provide valuable information about the abnormal neuroanatomy of aMCI, and also highlight a potential connection between structural changes and plasma Mir107 and BACE1 mRNA.

\section{MATERIALS AND METHODS}

\section{Participants}

This study was registered as a clinical trial with ClinicalTrials.gov registry number as NCT01819545 ${ }^{3}$. We recruited 20 patients with aMCI from the Shanghai Mental Health Center, Shanghai Jiao Tong University School of Medicine, and 24 cognitively normal elderly subjects from the Shanghai Changning District. Patients were enrolled by the hospital via self-referral or the referral from family or physician. This study was approved by the Institution's Ethical Committee of Shanghai Mental Health Center, Shanghai Jiao Tong University School of Medicine, and written informed consent was obtained from all subjects and/or their legal guardians. All experiments were performed in accordance with relevant guidelines and regulations.

aMCI was diagnosed based on the previously published criteria (McKhann et al., 2011). We amended the aMCI diagnostic criteria of the Petersen Mini Mental States Examination (MMSE; Folstein et al., 1975) in order to accommodate the low level of education in elderly Chinese. In the current study, we used revised MMSE cut-off scores as one of the criteria to recruit individual subjects (Katzman et al., 1988). The cognitively normal elderly control subjects (NC) were also recruited. They were independently functioning community dwellers with no neurological or psychiatric conditions.

All participants underwent a screening process that included a review of their medical history, physical and neurological examinations, laboratory tests, and MRI scans. The clinical assessment of MCI or dementia included neuropsychological tests, as well as behavioral and psychiatric interviews conducted by the attending psychiatrists. MMSE and Montreal Cognitive Assessment (MoCA; Nasreddine et al., 2005) were assessed in all participants. Based on assessment, aMCI patients were retained, and others who had impairments in a single non-memory domain or impairment in two or more domains were excluded.

\section{MR Image Acquisition and Processing}

MRI images were scanned with a Siemens MAGNETOM VERIO 3T scanner (Siemens, Munich). T1-weighted images were

\footnotetext{
${ }^{3}$ www.clinicaltrials.gov
} 


\begin{tabular}{|c|c|c|c|}
\hline Gene & Primer sequence & $\begin{array}{c}\text { Annealing } \\
\text { temperature } \\
\left({ }^{\circ} \mathrm{C}\right)\end{array}$ & $\begin{array}{l}\text { Lengths of } \\
\text { PCR } \\
\text { products (bp) }\end{array}$ \\
\hline $\begin{array}{l}\text { BACE1 } \\
\text { mRNA }\end{array}$ & $\begin{array}{l}\text { F:5'AAGTTCATTACCTCCCTATCAGT3' } \\
\text { R:5'AGGCCCTCCTTGTATTTCC3' }\end{array}$ & 60 & 186 \\
\hline Mir107 & $\begin{array}{l}\text { GSP:5'GCAGCAGCATTGTACAGG3' } \\
\text { R:5'CAGTGCGTGTCGTGGAGT3' }\end{array}$ & 60 & 65 \\
\hline
\end{tabular}

obtained with 128 sagittal slices using the 3D magnetization prepared rapid acquisition gradient echo sequence with the following parameters: $\mathrm{TR}=2530 \mathrm{~ms}, \mathrm{TE}=3.39 \mathrm{~ms}$, flip angle $=7^{\circ}$, spatial resolution $=1 \times 1 \times 1.3 \mathrm{~mm}^{3}$, and the acquisition time was 8 min 7 s. The MRI FLAIR data acquisition setting used the following parameters: matrix $256 \times 192, \mathrm{NEX}=1, \mathrm{FOV}=24 \mathrm{~cm}, \mathrm{TE}=140, \mathrm{TR}=8600$, InVTime $=2200$

Surface anatomy was extracted from MR images using FreeSurfer software package (version 5.3.0) ${ }^{4}$ (Fischl and Dale, 2000). We reviewed all obtained cortical surfaces and minimal manual editing was also performed at inaccurately segmented locations. The generated cortical surfaces were validated by comparing them with manual measures on MRI data (Fischl and Dale, 2000). CT was computed as the average distance between gray-white surface and pial surface. SA for each vertex was calculated on the pial surface, representing the area of the tessellated triangles linked to the vertex. The local cortical folding for each vertex was measured with LGI, which accounted for the ratio of local SA to the outer hull layer that tightly wrapped the pial surface. The folding was extended from two-dimensional gyrification measurement (Schaer et al., 2008).

\section{Blood Plasma Collection}

Blood samples were obtained from each subject and were centrifuged for $20 \mathrm{~min}$ at $4^{\circ} \mathrm{C}$ at $3000 \mathrm{rpm}$. A $200 \mu \mathrm{l}$ plasma aliquot was taken from each sample and immediately frozen and stored at $-80^{\circ} \mathrm{C}$.

\section{Quantitative RT-PCR (qRT-PCR)}

Total mRNA and miRNA were extracted using TRIZOL $^{\circledR}$ Reagent (Invitrogen, Carlsbad, CA, USA), and were quantified using a NanoDrop ${ }^{\circledR}$ ND-1000 spectrophotometer (Waltham, MA, USA). Total mRNA was subjected to qRT-PCR using $2 \times$ PCR master mix (Super Array, Valencia, CA, USA) and the ABI PRISM7900 system (Applied Biosystems, Foster City, CA, USA). For each sample, real-time PCR was performed for the target mRNA (BACE1; Table 1) together with the reference gene $\beta$-actin. The relative expression of the target mRNA was determined by the $2^{\Delta \Delta C T}$ method.

\footnotetext{
${ }^{4}$ http://surfer.nmr.mgh.harvard.edu/
}

Total miRNA qRT-PCR was conducted using the Universal cDNA Synthesis Kit (Exiqon, Vedbaek, Denmark) and the Gene Amp PCR System 9700 (Applied Biosystems, Foster City, CA, USA). Similarly, for each sample, real-time PCR was performed for the target Mir107 (Table 1) together with the reference gene microRNA423-5p. The relative expression of the target miRNA was determined by the $2^{\Delta \Delta C T}$ method.

\section{BACE1 Protein Concentration}

We used a BACE1 protein ELISA kits (LBL ${ }^{\circledR} 27752$ human BACE1 assay kit, Takasaki-Shi, Gunma, Japan) to assay the BACE1 protein concentration from our study participants.

\section{Statistical Analysis}

SPSS 17.0 was used for statistical analysis. Two-tailed $t$-tests were used to compare demographic characteristics between groups. Chi-square tests were used to measure differences in gender distribution. For each vertex, a general linear model was used to detect significant differences in CT, SA, and LGI between aMCI and controls patients, respectively. The confounding factors were regressed, including age, gender, education, and overall measurement. A smoothing kernel of $10 \mathrm{~mm}$ was applied before group comparison at the level of each vertex. Mir107, BACE1 mRNA, and BACE1 protein expression between groups were analyzed by two-tailed $t$-tests. Pearson correlation was used to examine correlations between plasma Mir107 and BACE1 mRNA expression and cortical anatomy.

\section{RESULTS}

\section{Demographic and Clinical Variables}

The demography and clinical scores for the aMCI group and the NC group are listed in Table 2. No significant differences between the groups were observed in age, gender or education, so the effects of age, gender, education level, and brain size were removed in our analysis. As expected, there were group differences in the MMSE, MoCA and Clinical Dementia Rating-Sum of Box (CDR-SOB) scores.

\section{Mir107, BACE1 mRNA, and Protein Level in Plasma}

Plasma levels of Mir107 and BACE1 mRNA were significantly different between aMCI and NC (Table 2). There were significant negative correlations between plasma Mir107 and BACE1 mRNA gene expression in aMCI and NC (Table 2). We did not find any significant difference in the plasma level of BACE1 protein between aMCI and NC subjects (Table 2).

\section{Cortical Thickness}

Samples from patients with aMCI showed widespread thinning of $\mathrm{CT}$ as compared to $\mathrm{NC}$ in the memory-associated cortical 
TABLE 2 | Demography, clinical scores, RT-PCR of Mir107, BACE1 mRNA, and protein expression in plasma of the subjects in the study.

\begin{tabular}{|c|c|c|c|c|c|c|}
\hline & \multirow[b]{2}{*}{$\operatorname{aMCl}(n=20)$} & \multirow[b]{2}{*}{ NC $(n=24)$} & \multirow[b]{2}{*}{$p$-value } & \multirow[b]{2}{*}{ Mean difference } & \multicolumn{2}{|c|}{$95 \% \mathrm{Cl}$ of the Mean difference } \\
\hline & & & & & Lower & Upper \\
\hline Age (years) & $70.1 \pm 7.2$ & $69.9 \pm 7.6$ & 0.81 & 0.18 & -4.34 & 4.7 \\
\hline Male/Female & $9 / 11$ & $13 / 11$ & 0.55 & - & - & \\
\hline Education (years) & $7.8 \pm 4.9$ & $9.4 \pm 4.1$ & 0.91 & -1.58 & -4.32 & 1.17 \\
\hline MMSE & $24.5 \pm 3.4$ & $27.8 \pm 2.8$ & $<0.001$ & -3.25 & -4.96 & -3.25 \\
\hline MoCA & $18.3 \pm 4.3$ & $24.0 \pm 3.6$ & $<0.001$ & -5.73 & -8.2 & -3.25 \\
\hline CDR-SOB & $2.0 \pm 0.7$ & $0.0 \pm 0.0$ & $<0.001$ & 2 & 1.67 & 2.34 \\
\hline Mir10 $7^{1}$ & $2.33 \pm 2.24$ & $3.85 \pm 1.24$ & 0.007 & -1.52 & -2.6 & -0.44 \\
\hline BACE1mRNA2 ${ }^{2}$ & $1.25 \pm 0.50$ & $0.88 \pm 0.37$ & 0.007 & 0.37 & 0.11 & 0.63 \\
\hline BACE1 (LINE) & $3.95 \pm 1.82$ & $3.22 \pm 1.07$ & 0.106 & 0.73 & -0.16 & 1.62 \\
\hline BACE1 (LOG) & $4.91 \pm 1.85$ & $4.17 \pm 1.25$ & 0.122 & 0.74 & -0.21 & 1.69 \\
\hline$r(1$ and 2$)$ & -0.761 & -0.648 & - & - & - & - \\
\hline$p$-value (1 and 2$)$ & $<0.001$ & 0.001 & - & - & - & - \\
\hline
\end{tabular}

Data represent mean $\pm S D$ and were analyzed by $t$-test; the $p$-values were determined from two-tailed t-tests except for gender, which was analyzed using the Chi squared test; 1 = Mir107, 2 = BACE1 mRNA, Pearson Correlation analysis; Symbol: - denotes Not Applicable.

areas. CT was thinner in the superior parietal gyrus, postcentral gyrus, lingual gyrus, and paracentral gyrus, compared to other brain regions both in aMCI and NC subjects (Figure 1A). There was significant difference within the aMCI patients with increased age $(p=0.014)$, but similar changes were not seen in the NC subjects (Figure 1B). Left brain areas associated with episodic memory and language showed the largest differences $(p<0.05)$ between the groups in left postcentral gyrus, left inferior parietal gyrus, left precuneus, and the upside right supramarginal gyrus. In addition, the left superior temporal gyrus and insula, as well as the low side right supramarginal fusiform gyrus, were significantly thicker in aMCI $(p<0.05$, Figure 1C).

\section{Cortical Surface Area}

There was no significant change between two groups in total SA, but aMCI patients showed reduced SA with the increased age when compared to NC. SA was reduced in the superior parietal gyrus, postcentral gyrus, middle temporal gyrus, and anterior cingulate gyrus in general (Figure 2A). There was no significant difference between aMCI and NC with the increased age (Figure 2B). ROI-based group analysis found the significantly reduced SA in left superior frontal gyrus, right supramarginal and caudal middle frontal gyrus, and right posterior cingulate gyrus in aMCI $(p<0.05)$. In addition, left postcentral gyrus had the significantly larger SA in aMCI $(p<0.01$, Figure 2C).

\section{Local Gyrification Index}

The aMCI group had significantly lower LGI in general. LGI was higher in the superior temporal gyrus and middle temporal gyrus than other brain regions in both groups (Figure 3A). aMCI showed reduced LGI with increase of age; a significant difference was found between groups $(p=0.038)$. There was also a significant decrease with the increased age within aMCI ( $p=0.046$, Figure 3B). The ROI-based LGI group analysis showed a significant effect in the right superior temporal gyrus $(p<0.05)$ in aMCI (Figure 3C).

\section{Abnormal Surface Anatomy, Neuropsychological Scores, and Plasma Mir107 and BACE1}

Changes in CT in aMCI were correlated with neuropsychological scores and plasma Mir107 expression (Figure 4A). Left postcentral gyrus CT was correlated with MMSE and MoCA. Left inferior parietal gyrus and precuneus cortex CT were correlated with MMSE and MoCA, as well as plasma Mir107. Right superior supramarginal gyrus CT was correlated with MMSE, while the right inferior supramarginal gyrus was negatively correlated with MoCA. Right fusiform gyrus CT was negatively correlated with MMSE and MoCA (Figure 4A).

Changes in aMCI SA were also correlated with neuropsychological test scores and plasma Mir107 and BACE1 mRNA expression (Figure 4B). SA of the left superior frontal gyrus was positively correlated with plasma Mir107 expression and negatively correlated to plasma BACE1 mRNA expression. SA of the right supramarginal gyrus, right caudal middle frontal gyrus, and right posterior cingulate cortex were positively correlated to MoCA (Figure 4B). We also found significant reduction in LGI in the right superior temporal gyrus in aMCI, which was correlated with MMSE $(p=0.009)$ and MoCA $(p=0.028$, Figure 4C).

\section{DISCUSSION}

The main goal of this study is to investigate changes in cortical anatomy in aMCI patients, especially on CT, SA, and LGI, and also to determine any correlation between structural changes and Mir107 and BACE1 plasma levels. The results obtained demonstrate a correlation between CTs of left precuneus cortex and left inferior parietal gyrus and plasma levels of Mir107, and between SA of left superior frontal gyrus and plasma levels of Mir107 and BACE1 mRNA. We hypothesize that the left precuneus cortex, left inferior parietal gyrus, and left superior frontal gyrus are the areas first damaged in 
A Mean cortical thickness ( $\mathrm{mm})$ in $\mathrm{aMCl}$ and $\mathrm{NC}$

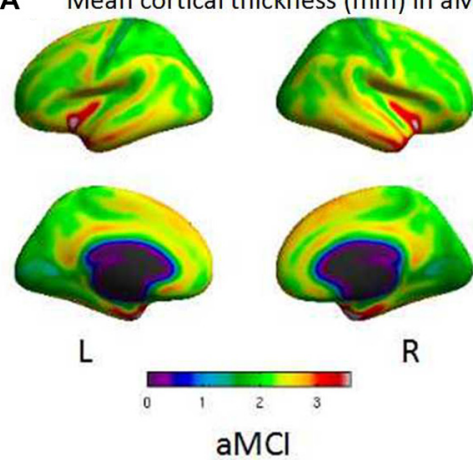

B Mean cortical thickness $(\mathrm{mm})$ as a function of age in $\mathrm{aMCl}$ and $\mathrm{NC}$

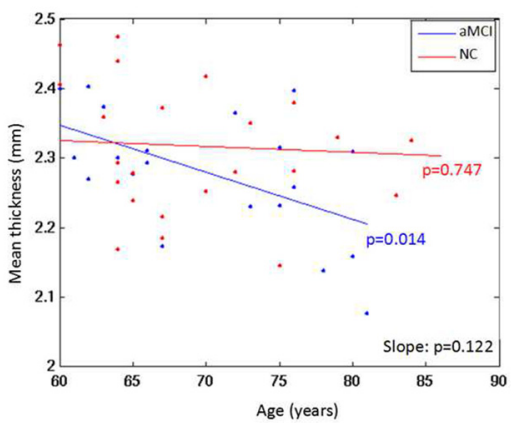

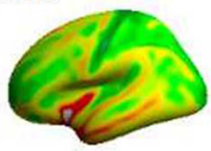
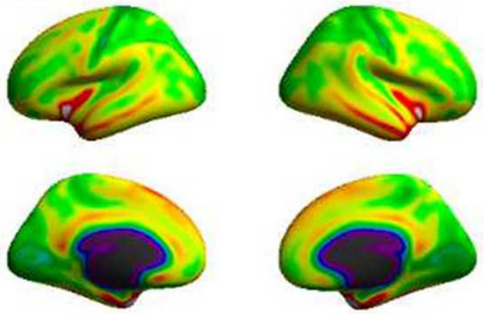

$\mathrm{L}$

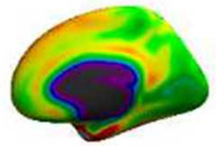

$\mathrm{R}$

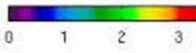

NC

C Brain regions with significantly different $\mathrm{CT}$ between $\mathrm{aMCl}$ and $\mathrm{NC} . \mathrm{p}<0.05$ with FDR correction and cluster size $>200$

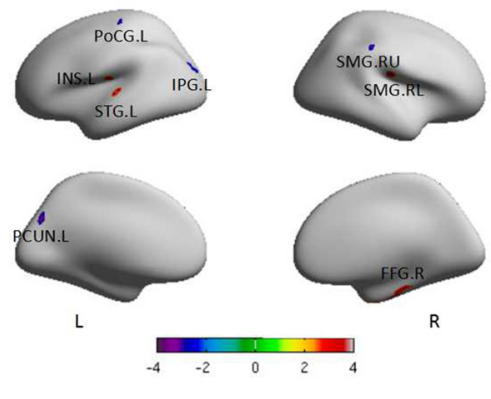

FIGURE 1 | (A) The cortex thicknesses are thinner in the superior parietal gyrus, postcentral gyrus, lingual gyrus and paracentral gyrus than other brain regions both in amnestic mild cognitive impairment (aMCl) and normal control (NC) in general. (B) aMCl shows less cortical thickness (CT) than NC when age increases but no significant difference is found between groups $(p=0.122)$. There is significant difference within group of aMCl when age increases $(p=0.014)$, while there is no significant difference within group of NC when age increases $(p=0.747)$. (C) The ROI-based analysis of CT revealed that the left postcentral gyrus (PoCG.L), the left inferior parietal gyrus (IPG.L), the left precuneus (PCUN.L) and the upside right supramarginal gyrus (SMG.RU) were significant group differences (p < 0.05) between the $\mathrm{aMCl}$ and the $\mathrm{NC}$ groups with the aMCl having thinner cortex than the NC. In addition, the left superior temporal gyrus (STG.L), the left insula (INS.L), the low side right supramarginal gyrus (SMG.RL) and the right fusiform gyrus (FFG.R) exhibited significantly $(p<0.05)$ larger thickness in the aMCl compared with the NC.

aMCI, and form the core regions of AD impairment. We also hypothesize that Mir107 is critical to the structural changes in $\mathrm{AD}$ brain cortex that are associated with cognition, language, and emotion.

\section{Alterations in Cortical Thickness}

We found that CT, especially the CT of left heteromodal association, was thinner in aMCI patients than in NC. CT is impacted by proliferation of myelin, a reduction in neuronal size or number, and changes in synapses (Sowell et al., 2004). CT is also influenced by the number and size of cells within a column, packing density, and by the extent of myelination (Eickhoff et al., 2005). Thus, alterations in CT may reflect abnormality in the underlying cell counts and organization.

Decreased CT in the left postcentral gyrus, left inferior parietal gyrus, and left precuneus cortex found in this study is in line with previous studies of aMCI, in which late aMCI showed cortical thinning in the bilateral dorsolateral prefrontal, anterior and medial temporal, and temperoparietal association cortices, as well as the precuneus (Ye et al., 2014). We found increased cortex thickness in the left superior temporal gyrus cortex, left insula, low side right supramarginal gyrus, and right fusiform gyrus in aMCI. These areas are associated with social cognition processes and word recognition. Some compensations probably occur in the onset of early AD stage.

Some CT results suggest that thinning of heteromodal association cortices is an early biomarker of $\mathrm{AD}$, not hippocampal loss (Sabuncu et al., 2011). We did not find significant hippocampal volume loss in aMCI; instead, we found changes in the heteromodal association cortices, such as left inferior parietal gyrus and left precuneus cortex. Researchers have also reported that $\mathrm{AD}$ patients had the reduced $\mathrm{CT}$ bilaterally in the precuneus, which is characteristic of $\mathrm{AD}$ pathology (Watson et al., 2015). The precuneus is important for the recall of episodic memories, and is involved in source memory with the left inferior prefrontal cortex (Sadigh-Eteghad et al., 2014). The role of the precuneus is suggested to provide the rich episodic contextual associations used by the prefrontal gyrus to select correct memories (Sadigh-Eteghad et al., 2014). In recollection, the precuneus seems to determine whether context information exists, and therefore could be involved in diverse processes as episodic memory retrieval, working memory, and conscious perception. 

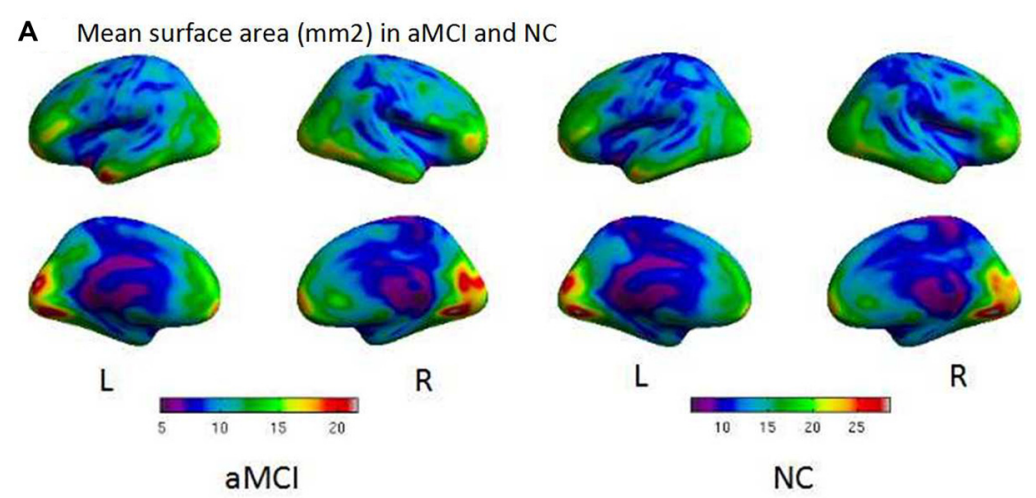

B Mean surface area $(\mathrm{mm} 2)$ as a function of age in $\mathrm{aMCl}$ and $\mathrm{NC}$

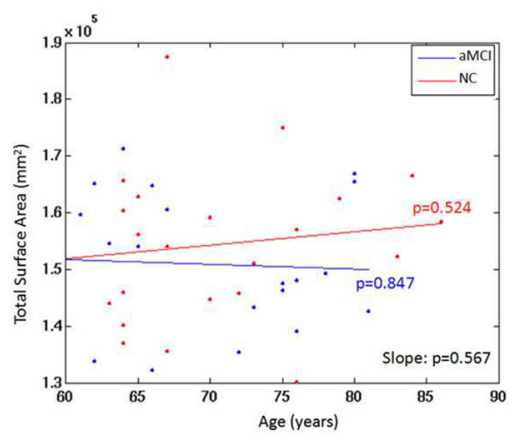

C Brain regions with significantly different $\mathrm{SA}$ between $\mathrm{aMCl}$ and $\mathrm{NC} . \mathrm{p}<0.05$ with FDR correction and cluster size $>200$

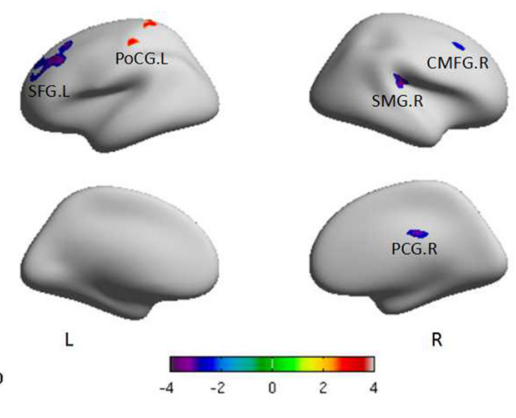

FIGURE 2 | (A) There is no significant difference between two groups on the total surface area (SA). But aMCl shows less SA than NC when age increases. The surface areas are less in the superior parietal gyrus, postcentral gyrus, middle temporal gyrus and anterior cingulate gyrus than other brain regions both in aMCl and NC in general. (B) aMCl shows less SA than NC when age increases but no significant difference is found between groups $(p=0.567)$. There are no significant difference within group of aMCI $(p=0.847)$ and $\mathrm{NC}(p=0.524)$ when age increases, respectively. (C) ROI based group analysis found significantly $(p<0.05)$ smaller SA in the left superior frontal gyrus (SFG.L), the right supramarginal gyrus (SMG.R), the right caudal middle frontal gyrus (CMFG.R) and the right posterior cingulate gyrus (PCG.R) in the aMCl, compared with the NC. In addition, the left postcentral gyrus (PoCG.L; $p<0.01$ ) exhibited significantly larger SA in the aMCl compared with the NC.

Our findings are consistent with the Pittsburgh Compound-B (PIB) and Fluorodeoxyglucose-Positron Emission Tomography (FDG-PET) imaging studies. For example, AD patients showed significant PIB retention in the bilateral precuneus, temporal lobe, and cingulate gyrus, as well as glucose hypometabolism in these areas as well as the inferior parietal and middle frontal gyrus, left precentral and parahippocampal gyrus, right superior frontal gyrus, and thalamus (He et al., 2015). The results seen in our study are in line with previous studies of aMCI. We conclude from these findings that structural changes may occur at the onset of aMCI, and can be detected by CT assessment.

\section{Alterations in Surface Area}

We found reduced SA in the left superior frontal gyrus, right supramarginal gyrus, right caudal middle frontal and right posterior cingulate gyrus in aMCI. A few prior studies have examined SA in aMCI with rather inconsistent results. The $\mathrm{SA}$ regions implicated in aMCI and NC include the superior temporal gyrus, lingual gyrus, and superior frontal gyrus in the left hemisphere, as well as the angular gyrus, lateral orbitofrontal sulcus, inferior parietal sulcus, middle frontal rostral region, pars triangularis gyrus, central sulcus, temporal pole, superior temporal sulcus, and the precentral gyrus in the right hemispheres (Li et al., 2014b). Another study found the SA in progressive aMCI was slightly greater than in $\mathrm{AD}$ (Liao et al., 2014), but with lower cortical GM surface areas in left superior temporal, supramarginal, and inferior parietal cortices (Madsen et al., 2015).

SA has been previously found to be strongly positively correlated with head size and brain size (Dickerson et al., 2009), and is also related to the number of minicolumns in cortex, as the SA of an area is driven by the number of columns. This is important considering the increased number of minicolumns in the frontal and temporal areas in aMCI (Casanova et al., 2010). Thus, alterations in SA in aMCI may be explained by abnormal minicolumn counts, or overall differences in brain size.

\section{Alterations in Cortical Folding}

Sulcal folds are the principal surface landmarks of the cerebral cortex, and exhibit structurally complex patterns that are 


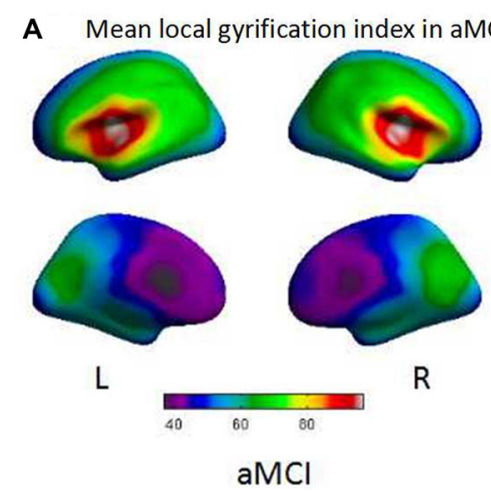

B Mean local gyrification index as a function of age in $\mathrm{aMCl}$ and $\mathrm{NC}$

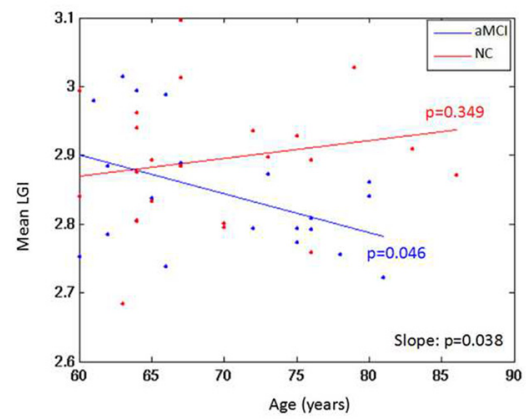

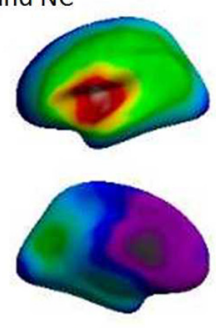

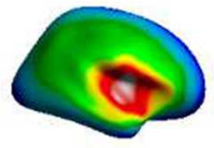

$\mathrm{L}$

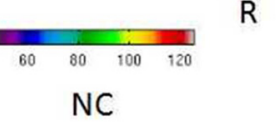

C Brain regions with significantly different LGI between $\mathrm{aMCl}$ and $\mathrm{NC} . \mathrm{p}<0.05$ with FDR correction and cluster size $>200$

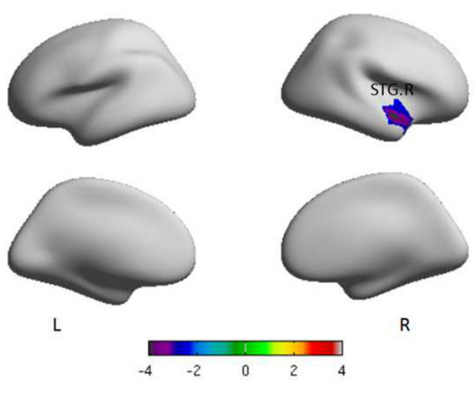

FIGURE 3 | (A) Compared with controls, aMCl has significant difference on the general local gyrification index (LGI) and one cluster where aMCI has significant LGI reduction. The LGl are higher in the superior temporal gyrus, and middle temporal gyrus than other brain regions both in aMCl and $\mathrm{NC}$ in general. (B) aMCl shows less CT than NC when age increases and significant difference is found between groups $(p=0.038)$. There is significant difference within group of aMCl when age increases $(p=0.046)$, while there is no significant difference within group of NC when age increases $(p=0.349)$. (C) The ROI based LGl group analysis shows a significant effect of the right superior temporal gyrus (STG.R; $p<0.05$ ) in aMCl.

postulated to reflect underlying connectivity (Van Essen, 1997). Changes in folding geometry have been shown to develop with aging and to be associated with cognitive decline (Kochunov et al., 2005; Liu et al., 2011). Sulcal of individuals with MCI and $\mathrm{AD}$ had less curvature and depth than those of cognitively NCs (Im et al., 2008). These differences were observed to be the largest in the temporal lobe. Cognitive functioning, as assessed by MMSE scores, decreased as global cortex gyrification decreased. Abnormalities of global cortex gyrification and regional sulcal span are characteristic of patients with even mild AD and they may thus facilitate early diagnosis of this condition (Liu et al., 2012).

There are reports of mild AD patients with lower gyrification index than controls (King et al., 2010). A consistent pattern of gyrification changes was seen also in dementia subjects, with regions generally affected early in the progression of AD pathology for decreased gyrification (Lebed et al., 2013). We found a reduction in global gyrification in aMCI. A previous study found sulcal widening in $\mathrm{AD}$ in the frontal, parietal, temporal, and occipital lobes (Im et al., 2008). Our present study found AD-associated widening of individual sulcal within brain lobes, particularly in the superior frontal and superior temporal sulcal. We suspect the right superior temporal gyrus may be a contributor to the $\mathrm{AD}$ effects observed. The gyrification of right superior temporal gyrus in the controls and aMCI patients was reported to be positively associated with MMSE and MoCA scores, supporting previous associations between cognitive performance and cortical morphology in elderly individuals (Liu et al., 2011).

\section{Plasma Levels of Mir107 and BACE1 mRNA and Abnormal Cortical Anatomy}

There was a significant change in Mir107 and BACE1 mRNA in the plasma samples from aMCI and NC subjects, suggesting that plasma Mir107 and BACE1 mRNA expression may be linked to the decrease in CT in the precuneus and cortical SA of the left superior frontal gyrus. Evidence has suggested that the alterations of miRNAs could contribute to AD risk (Hébert et al., 2010). The study has demonstrated that specific miRNA levels were altered in the brains, CSF, and plasma of $\mathrm{AD}$ patients (Hébert et al., 2010), raising the possibility of using these compartments for diagnosis.

A combination of genetic data and neuroimaging techniques is increasingly becoming a winning strategy in identifying 
A Cortex Thickness $(\mathrm{mm})$

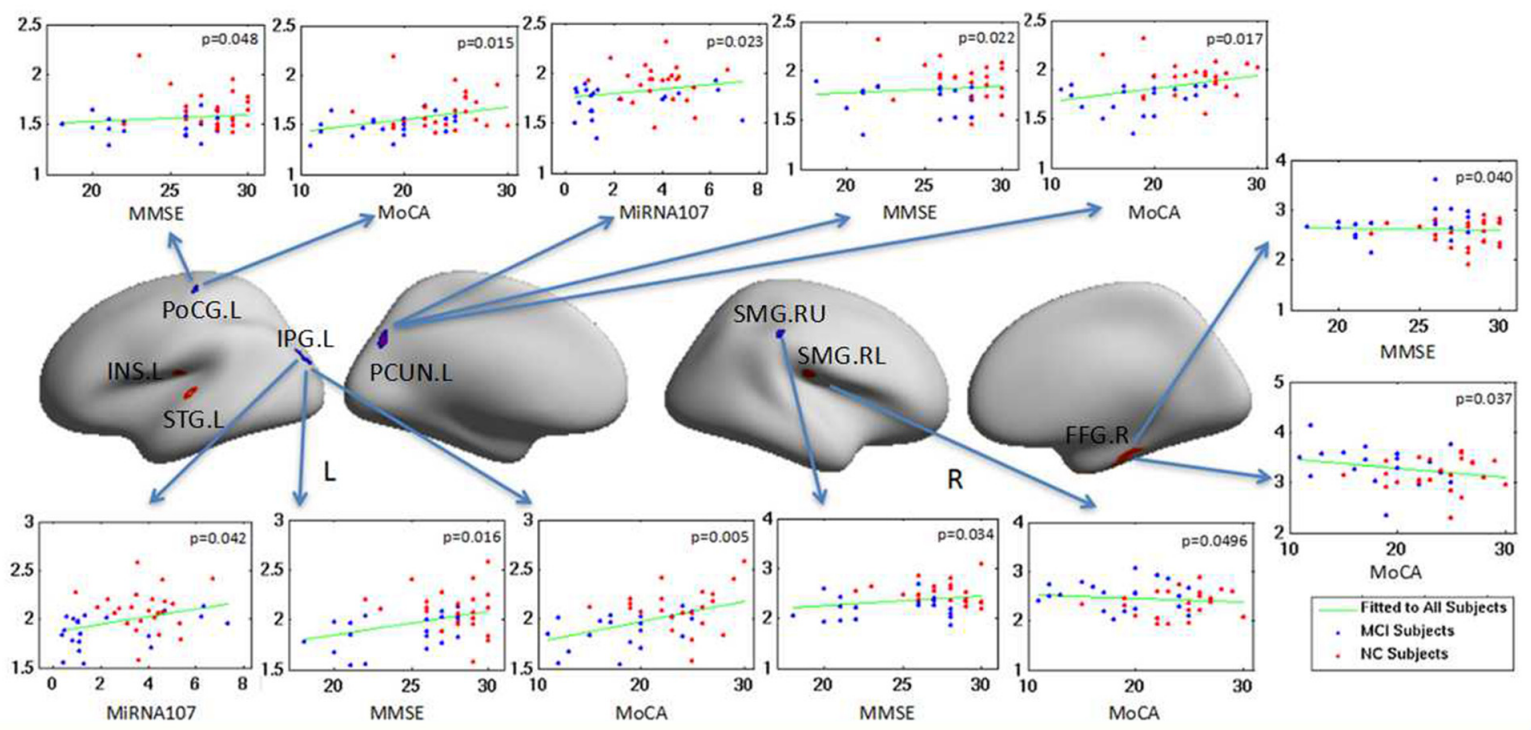

B Surface Area $\left(\mathrm{mm}^{2}\right)$

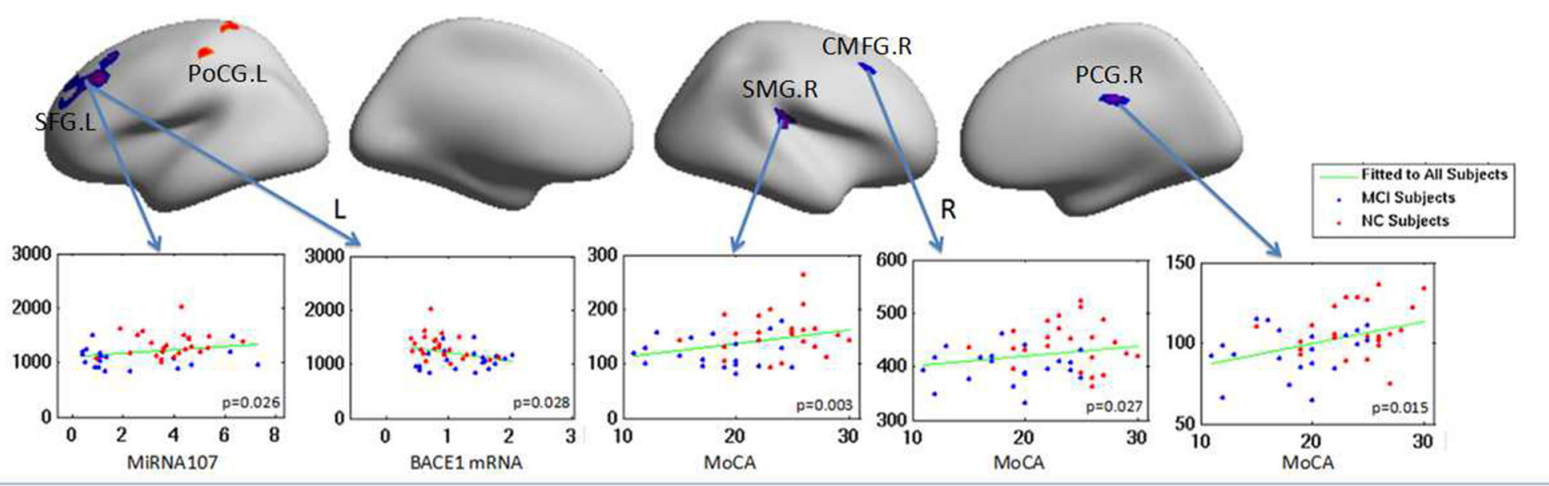

C Local Gyrification Index
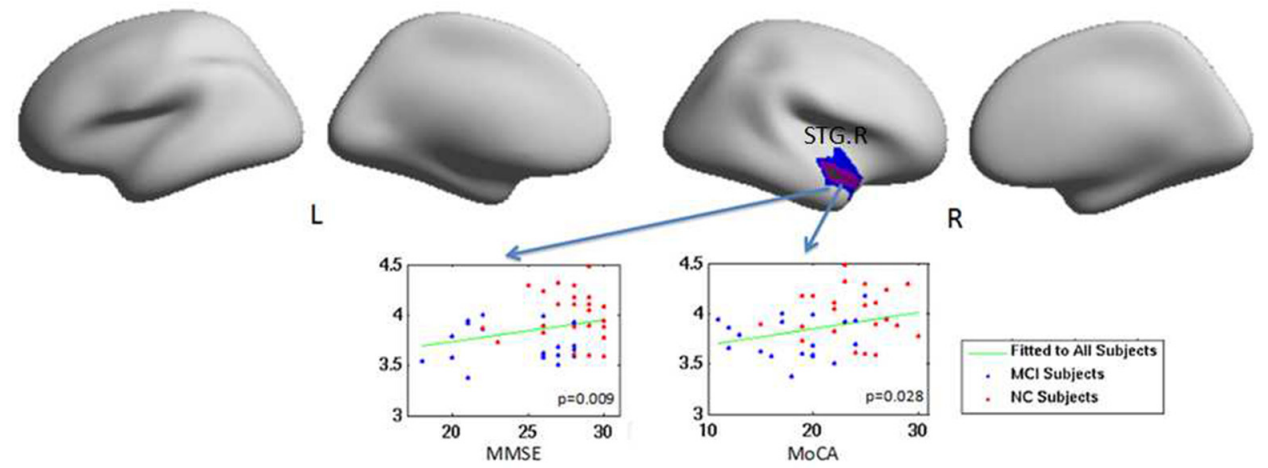

FIGURE 4 | The relationship between brain regions with abnormal surface anatomy in aMCl and neuropsychological test scores and the plasma target gene expressions. Changes in CT, SA and LGI in aMCl were correlated with neuropsychological scores and/or plasma MiRNA 107 expression. (A) The relationship of CT with neuropsycological test score and the plasma expression of MiRNA 107. (B) The relationship of SA with neuropsycological test score and the plasma expression of MiRNA 107 and BACE1 mRNA. (C) The relationship of local gyrification and neuropsycological test score.

preclinical stages of AD. By MRI, FDG-PET, and amyloid PET, characteristics of AD can be detected in clinically normal subjects who are genetically predisposed to the disease. In particular, presenilin-1 mutation carriers with aMCI have smaller brain volumes in the thalamus, splenium and pons, and originally involve with the left temporal lobe (Lee et al., 2013). 
Apolipoprotein E genotype and family history also have an independent and/or additive contribution to brain structural changes (Honea et al., 2010).

In this study, we demonstrate for that peripheral Mir107 and BACE1 mRNA levels were associated with abnormal brain cortical anatomy, including the decrease in CT of precuneus cortex and in SA of left superior frontal gyrus in aMCI patients. These finding suggest that peripheral Mir107 and BACE1 mRNA can be a candidate of early biomarkers for $\mathrm{AD}$ and may be important for the onset of $\mathrm{AD}$. We also found that the plasma level of Mir107 was altered in the same fashion as plasma BACE1 mRNA, suggesting that Mir107 may be central in the pathogenesis of $\mathrm{AD}$. This combination study of brain anatomical disruption and gene information, to our knowledge, has not been attempted previously and may shed light on our understanding of pathology of $\mathrm{AD}$ beyond amyloid protein theory. In the future, we will continue acquiring MRI scans with more subjects and will conduct a larger population study to further explore this topic.

\section{REFERENCES}

Albert, M. S., DeKosky, S. T., Dickson, D., Dubois, B., Feldman, H. H., Fox, N. C., et al. (2011). The diagnosis of mild cognitive impairment due to Alzheimer's disease: recommendations from the National Institute on Aging-Alzheimer's Association workgroups on diagnostic guidelines for Alzheimer's disease. Alzheimers Dement. 7, 270-279. doi: 10.1016/j.jalz.2011. 03.008

Apostolova, L. G., Steiner, C. A., Akopyan, G. G., Dutton, R. A., Hayashi, K. M., Toga, A. W., et al. (2007). Three-dimensional gray matter atrophy mapping in mild cognitive impairment and mild Alzheimer disease. Arch. Neurol. 64, 1489-1495. doi: 10.1001/archneur.64.10.1489

Bettens, K., Brouwers, N., Engelborghs, S., Van Miegroet, H., De Deyn, P. P., Theuns, J., et al. (2009). APP and BACE1 miRNA genetic variability has no major role in risk for Alzheimer disease. Hum. Mutat. 30, 1207-1213. doi: 10. 1002/humu.21027

Casanova, M. F., El-Baz, A., Vanbogaert, E., Narahari, P., and Switala, A. (2010). A topographic study of minicolumnar core width by lamina comparison between autistic subjects and controls: possible minicolumnar disruption due to an anatomical element in-common to multiple laminae. Brain Pathol. 20, 451-458. doi: 10.1111/j.1750-3639.2009.00319.x

Davatzikos, C. (2004). Why voxel-based morphometric analysis should be used with great caution when characterizing group differences. Neuroimage 23, 17-20. doi: 10.1016/j.neuroimage.2004.05.010

De Strooper, B. (2010). Proteases and proteolysis in Alzheimer disease: a multifactorial view on the disease process. Physiol. Rev. 90, 465-494. doi: 10. 1152/physrev.00023.2009

Dickerson, B. C., Bakkour, A., Salat, D. H., Feczko, E., Pacheco, J., Greve, D. N., et al. (2009). The cortical signature of Alzheimer's disease: regionally specific cortical thinning relates to symptom severity in very mild to mild $\mathrm{AD}$ dementia and is detectable in asymptomatic amyloid-positive individuals. Cereb. Cortex 19, 497-510. doi: 10.1093/cercor/bhn113

Dislich, B., and Lichtenthaler, S. F. (2012). The membrane-bound aspartyl protease BACE1: molecular and functional properties in Alzheimer's disease and beyond. Front. Physiol. 3:8. doi: 10.3389/fphys.2012.00008

Eickhoff, S., Walters, N. B., Schleicher, A., Kril, J., Egan, G. F., Zilles, K., et al. (2005). High-resolution MRI reflects myeloarchitecture and cytoarchitecture of human cerebral cortex. Hum. Brain Mapp. 24, 206-215. doi: 10.1002/hbm. 20082

Fischl, B., and Dale, A. M. (2000). Measuring the thickness of the human cerebral cortex from magnetic resonance images. Proc. Natl. Acad. Sci. U S A. 97, 11050-11055. doi: 10.1073/pnas.200033797

\section{AUTHOR CONTRIBUTIONS}

TW, SX and DS designed the study. SX and DS were responsible for the study, supervised data collection, statistical analysis, and modified the manuscript. TW, FS and YJ carried out statistical analysis, and wrote the main manuscript text. TW, FS, YJ and WJ prepared Figures 1-4. All authors reviewed the manuscript.

\section{ACKNOWLEDGMENTS}

This work was partially supported by the National Natural Science Foundation of China (81571298, 81201030, 61210001 and 61473190), the Shanghai Science and Technology Committee grants (14411965000 and 134119a2600), the Shanghai Jiao Tong University technological innovation special fund (YG2014MS39), and the SHSMU-ION Research Center for Brain Disorders, and the Fly Program of SMHC. This work was also supported in part by National Institutes of Health (NIH) grants AG041721, EB006733, EB008374, and EB009634.

Fleisher, A. S., Chen, K., Quiroz, Y. T., Jakimovich, L. J., Gomez, M. G., Langois, C. M., et al. (2012). Florbetapir PET analysis of amyloid- $\beta$ deposition in the presenilin $1 \mathrm{E} 280 \mathrm{~A}$ autosomal dominant Alzheimer's disease kindred: a cross-sectional study. Lancet Neurol. 11, 1057-1065. doi: 10.1016/S14744422(12)70227-2

Folstein, M. F., Folstein, S. E., and McHugh, P. R. (1975). “Mini-mental state”. A practical method for grading the cognitive state of patients for the clinician. J. Psychiatr. Res. 12, 189-198. doi: 10.1007/springerreference_61498

He, W., Liu, D., Radua, J., Li, G., Han, B., and Sun, Z. (2015). Metaanalytic comparison between PIB-PET and FDG-PET results in Alzheimer's disease and MCI. Cell Biochem. Biophys. 71, 17-26. doi: 10.1007/s12013-0140138-7

Hébert, S. S., Papadopoulou, A. S., Smith, P., Galas, M. C., Planel, E., Silahtaroglu, A. N., et al. (2010). Genetic ablation of Dicer in adult forebrain neurons results in abnormal tau hyperphosphorylation and neurodegeneration. Hum. Mol. Genet. 19, 3959-3969. doi: 10.1093/hmg/ddq311

Honea, R. A., Swerdlow, R. H., Vidoni, E. D., Goodwin, J., and Burns, J. M. (2010). Reduced gray matter volume in normal adults with a maternal family history of Alzheimer Disease. Neurology 74, 113-120. doi: 10.1212/wnl. 0b013e3181c918cb

Im, K., Lee, J. M., Seo, S. W., Hyung Kim, S., Kim, S. I., and Na, D. L. (2008). Sulcal morphology changes and their relationship with cortical thickness and gyral white matter volume in mild cognitive impairment and Alzheimer's disease. Neuroimage 43, 103-113. doi: 10.1016/j.neuroimage.2008. 07.016

Jin, Y., Shi, Y., Zhan, L., and Thompson, P. M. (2015). “Automated Multi-atlas labeling of the fornix and its integrity in Alzheimer's disease", in Proceedings of IEEE International Symposium Biomedical Imaging, (New York), 140-143.

Kandimalla, R. J., Prabhakar, S., Wani, W. Y., Kaushal, A., Gupta, N. Sharma, D. R., et al. (2013b). CSF p-Tau levels in the prediction of Alzheimer's disease. Biol. Open., 2:1119-1124. doi: 10.1242/bio.20135447

Kandimalla, R. J., Wani, W. Y., Anand, R., Kaushal, A., Prabhakar, S., Grover, V. K., et al. (2013a). Apolipoprotein E levels in the cerebrospinal fluid of north Indian patients with Alzheimer's disease. Am. J. Alzheimers Dis. Other Demen. 28, 258-262. doi: 10.1177/1533317513481097

Katzman, R., Zhang, M. Y., Ouang-Ya-Qu, Wang, Z. Y., Liu, W. T., Yu, E., et al. (1988). A Chinese version of the Mini-Mental State Examination; impact of illiteracy in a Shanghai dementia survey. J. Clin. Epidemiol. 41, 971-978. doi: 10. 1007/springerreference_61498

King, R. D., Brown, B., Hwang, M., Jeon, T., and George, A. T. (2010) Fractal dimension analysis of the cortical ribbon in mild Alzheimer's disease. Neuroimage 53, 471-479. doi: 10.1016/j.neuroimage.2010.06.050 
Kochunov, P., Mangin, J. F., Coyle, T., Lancaster, J., Thompson, P., Rivière, D., et al. (2005). Age-related morphology trends of cortical sulci. Hum. Brain Mapp. 26, 210-220. doi: 10.1002/hbm.20198

Lebed, E., Jacova, C., Wang, L., and Beg, M. F. (2013). Novel surface-smoothing based local gyrification index. IEEE Trans. Med. Imaging 32, 660-669. doi: 10. 1109/tmi.2012.2230640

Lee, G. J., Lu, P. H., Medina, L. D., Rodriguez-Agudelo, Y., Melchor, S., Coppola, G., et al. (2013). Regional brain volume differences in symptomatic and presymptomatic carriers of familial Alzheimer's disease mutations. J. Neurol. Neurosurg. Psychiatry 84, 154-162. doi: 10.1136/jnnp-2011302087

Li, J., Jin, Y., Shi, Y., Dinov, I. D., Wang, D. J., Toga, A. W., et al. (2013). Voxelwise spectral diffusional connectivity and its application to Alzheimer's disease and intelligence prediction. Med. Image Comput. Comput. Assist. Interv. 8149, 655-662. doi: 10.1007/978-3-642-40811-3_82

Li, G., Wang, L., Shi, F., Lyall, A. E., Lin, W., Gilmore, J. H., et al. (2014a). Mapping longitudinal development of local cortical gyrification in infants from birth to 2 years of age. J. Neurosci. 34, 4228-4238. doi: 10.1523/jneurosci.397613.2014

Li, S., Yuan, X., Pu, F., Li, D., Fan, Y., Wu, L., et al. (2014b). Abnormal changes of multidimensional surface features using multivariate pattern classification in amnestic mild cognitive impairment patients. J. Neurosci. 34, 10541-10553. doi: 10.1523/jneurosci.4356-13.2014

Liao, W., Long, X., Jiang, C., Diao, Y., Liu, X., Zheng, H., et al. (2014). Discerning mild cognitive impairment and Alzheimer disease from normal aging: morphologic characterization based on univariate and multivariate models. Acad. Radiol. 21, 597-604. doi: 10.1016/j.acra.2013.12.001

Libero, L. E., DeRamus, T. P., Deshpande, H. D., and Kana, R. K. (2014). Surfacebased morphometry of the cortical architecture of autism spectrum disorders: volume, thickness, area and gyrification. Neuropsychologia 62, 1-10. doi: 10 . 1016/j.neuropsychologia.2014.07.001

Liu, T., Lipnicki, D. M., Zhu, W., Tao, D., Zhang, C., Cui, Y., et al. (2012). Cortical gyrification and sulcal spans in early stage Alzheimer's disease. PLoS One 7: e31083. doi: 10.1371/journal.pone.0031083

Liu, T., Wen, W., Zhu, W., Kochan, N. A., Trollor, J. N., Reppermund, S., et al. (2011). The relationship between cortical sulcal variability and cognitive performance in the elderly. Neuroimage 56, 865-873. doi: $10.1016 / \mathrm{j}$. neuroimage.2011.03.015

Madsen, S. K., Rajagopalan, P., Joshi, S. H., Toga, A. W., Thompson, P. M., and the Alzheimer's Disease Neuroimaging Initiative (ADNI). (2015). Higher homocysteie associated with thinner cortical gray matter in 803 participants from the Alzheimer's Disease Neuroimaging Initiative. Neurobiol. Aging 36, S203-S210. doi: 10.1016/j.neurobiolaging.2014.01.154

McKhann, G. M., Knopman, D. S., Chertkow, H., Hyman, B. T., Jack, C. R., Kawas, C. H., et al. (2011). The diagnosis of dementia due to Alzheimer's disease: recommendations from the National Institute on Aging-Alzheimer's Association workgroups on diagnostic guidelines for Alzheimer's disease. Alzheimers Dement. 7, 263-269. doi: 10.1016/j.jalz.2011.03.005

Meda, S. A., Koran, M. E., Pryweller, J. R., Vega, J. N., Thornton-Wells, T. A., and the Alzheimer's Disease Neuroimaging Initiative (2013). Genetic interactions associated with 12-month atrophy in hippocampus and entorhinal cortex in Alzheimer's Disease Neuroimaging Initiative. Neurobiol. Aging 34, 1518.e9-1518.e18. doi: 10.1016/j.neurobiolaging.2012.09.020

Nasreddine, Z. S., Phillips, N. A., Bédirian, V., Charbonneau, S., Whitehead, V., Collin, I., et al. (2005). The Montreal Cognitive Assessment, MoCA: a brief screening tool for mild cognitive impairment. J. Am. Geriatr. Soc 53, 695-699. doi: 10.1111/j.1532-5415.2005.53221.x

Petersen, R. C., Parisi, J. E., Dickson, D. W., Johnson, K. A., Knopman, D. S., Boeve, B. F., et al. (2006). Neuropathology of amnestic mild cognitive impairment. Arch. Neurol. 63, 645-646. doi: 10.1001/archneur 63.5.645

Sabuncu, M. R., Desikan, R. S., Sepulcre, J., Yeo, B. T., Liu, H., Schmansky, N. J., et al. (2011). The dynamics of cortical and hippocampal atrophy in Alzheimer disease. Arch. Neurol. 68, 1040-1048. doi: 10.1001/archneurol. 2011.167

Sadigh-Eteghad, S., Majdi, A., Farhoudi, M., Talebi, M., and Mahmoudi, J. (2014). Different patterns of brain activation in normal aging and Alzheimer's disease from cognitional sight: meta analysis using activation likelihood estimation. J. Neurol. Sci. 343, 159-166. doi: 10.1016/j.jns.2014.05.066

Sathya, M., Premkumar, P., Karthick, C., Moorthi, P., Jayachandran, K. S., and Anusuyadevi, M. (2012). BACE1 in Alzheimer's disease. Clin. Chim. Acta. 414, 171-178. doi: 10.1016/j.cca.2012.08.013

Schaer, M., Cuadra, M. B., Tamarit, L., Lazeyras, F., Eliez, S., and Thiran, J. P. (2008). A surface-based approach to quantify local cortical gyrification. IEEE Trans. Med. Imaging. 27, 161-170. doi: 10.1109/tmi.2007.903576

Sowell, E. R., Thompson, P. M., Leonard, C. M., Welcome, S. E., Kan, E., and Toga, A. W. (2004). Longitudinal mapping of cortical thickness and brain growth in normal children. J. Neurosci. 24, 8223-8231. doi: 10.1523/jneurosci. 1798-04.2004

Van Essen, D. C. (1997). A tension-based theory of morphogenesis and compact wiring in the central nervous system. Nature 385, 313-318. doi: 10 . 1038/385313a0

Wang, T., Shi, F., Jin, Y., Yap, P.-T., Wee, C. Y., Zhang, J., et al. (2016). Multilevel deficiency of white matter connectivity networks in Alzheimer's disease: a diffusion MRI study with DTI and HARDI models. Neural Plast. 2016:2947136. doi: 10.1155/2016/2947136

Wang, W. X., Huang, Q., Hu, Y., Stromberg, A. J., and Nelson, P. T. (2011). Patterns of microRNA expression in normal and early Alzheimer's disease human temporal cortex: white matter versus gray matter. Acta. Neuropathol. 121, 193-205. doi: 10.1007/s00401-010-0756-0

Watson, R., Colloby, S. J., Blamire, A. M., and O'Brien, J. T. (2015). Assessment of regional gray matter loss in dementia with Lewy bodies: a surface-based MRI analysis. Am. J. Geriatr. Psychiatry 23, 38-46. doi: 10.1016/j.jagp.2014. 07.005

Ye, B. S., Seo, S. W., Yang, J. J., Kim, H. J., Kim, Y. J., Yoon, C. W., et al. (2014). Comparison of cortical thickness in patients with early-stage versus latestage amnestic mild cognitive impairment. Eur. J. Neurol. 21, 86-92. doi: 10. 1111/ene.12251

Zhan, L., Nie, Z., Ye, J., Wang, J., Jin, Y., Jahanshad, N., et al. (2014). “Multiple stages classification of Alzheimer's disease based on structural brain networks using generalized low rank approximation (GLRAM)," in Computational Diffusion MRI. Mathematics and Visualization. (Switzerland: Springer), 35-44.

Zhan, L., Zhou, J., Wang, Y., Jin, Y., Jahanshad, N., Prasad, G., et al. (2015). Comparison of nine tractography algorithms for detecting abnormal structural brain networks in Alzheimer's disease. Front. Aging Neurosci. 7:48. doi: 10. 3389/fnagi.2015.00048

Conflict of Interest Statement: The authors declare that the research was conducted in the absence of any commercial or financial relationships that could be construed as a potential conflict of interest.

Copyright (C) 2016 Wang, Shi, Jin, Jiang, Shen and Xiao. This is an open-access article distributed under the terms of the Creative Commons Attribution License (CC BY). The use, distribution and reproduction in other forums is permitted, provided the original author(s) or licensor are credited and that the original publication in this journal is cited, in accordance with accepted academic practice. No use, distribution or reproduction is permitted which does not comply with these terms. 